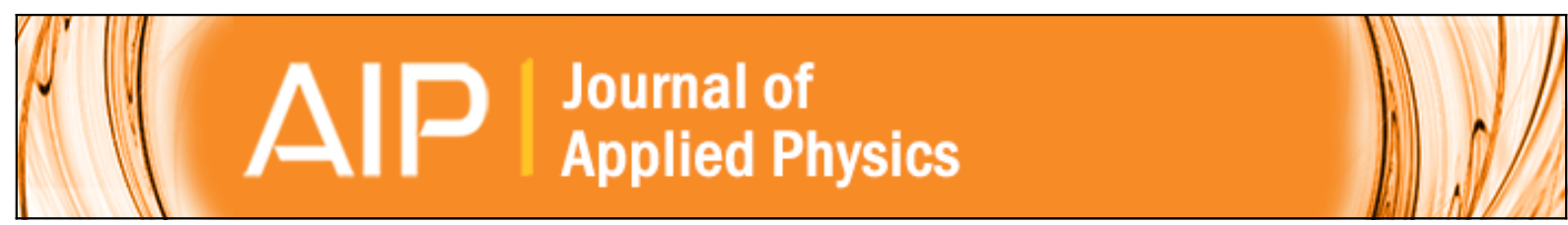

\title{
Local atomic order, electronic structure and electron transport properties of $\mathrm{Cu}-\mathrm{Zr}$
} metallic glasses

J. Antonowicz, A. Pietnoczka, K. Pękała, J. Latuch, and G. A. Evangelakis

Citation: Journal of Applied Physics 115, 203714 (2014); doi: 10.1063/1.4879903

View online: http://dx.doi.org/10.1063/1.4879903

View Table of Contents: http://scitation.aip.org/content/aip/journal/jap/115/20?ver=pdfcov

Published by the AIP Publishing

\section{Articles you may be interested in}

Energetics of local clusters in $\mathrm{Cu} 64.5 \mathrm{Zr} 35.5$ metallic liquid and glass

Appl. Phys. Lett. 97, 021901 (2010); 10.1063/1.3464164

The basic polyhedral clusters, the optimum glass formers, and the composition-structure-property (glass-forming ability) correlation in $\mathrm{Cu}-\mathrm{Zr}$ metallic glasses

J. Appl. Phys. 107, 063508 (2010); 10.1063/1.3359683

Statistical composition-structure-property correlation and glass-forming ability based on the full icosahedra in $\mathrm{Cu}-\mathrm{Zr}$ metallic glasses

Appl. Phys. Lett. 96, 061903 (2010); 10.1063/1.3310278

Al-centered icosahedral ordering in $\mathrm{Cu} 46 \mathrm{Zr} 46 \mathrm{Al} 8$ bulk metallic glass

Appl. Phys. Lett. 94, 091904 (2009); 10.1063/1.3086885

Icosahedral ordering in $\mathrm{Zr} 41 \mathrm{Ti} 14 \mathrm{Cu} 12.5 \mathrm{Ni} 10 \mathrm{Be} 22.5$ bulk metallic glass

Appl. Phys. Lett. 92, 201913 (2008); 10.1063/1.2931702

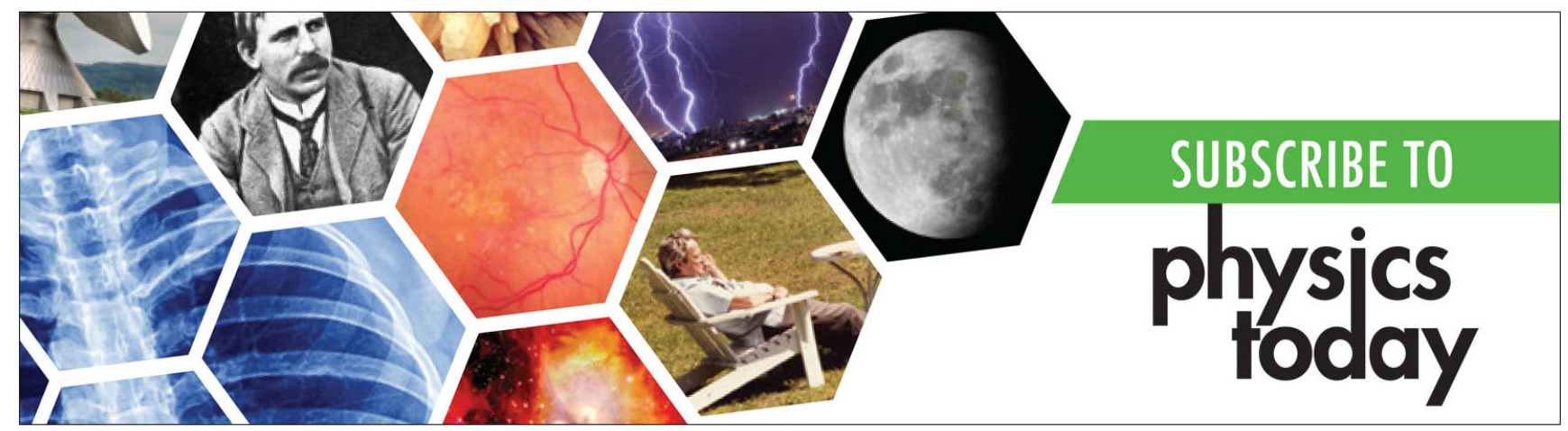




\title{
Local atomic order, electronic structure and electron transport properties of Cu-Zr metallic glasses
}

\author{
J. Antonowicz, ${ }^{1, a)}$ A. Pietnoczka, ${ }^{1}$ K. Peckała, ${ }^{1}$ J. Latuch, ${ }^{2}$ and G. A. Evangelakis ${ }^{3}$ \\ ${ }^{1}$ Faculty of Physics, Warsaw University of Technology, Koszykowa 75, 00-662 Warsaw, Poland \\ ${ }^{2}$ Faculty of Materials Science and Engineering, Warsaw University of Technology, Wołoska 141, \\ 02-507 Warsaw, Poland \\ ${ }^{3}$ Department of Physics, University of Ioannina, Ioannina 45110, Greece
}

(Received 10 February 2014; accepted 14 May 2014; published online 29 May 2014)

\begin{abstract}
We studied atomic and electronic structures of binary $\mathrm{Cu}-\mathrm{Zr}$ metallic glasses (MGs) using combined experimental and computational methods including X-ray absorption fine structure spectroscopy, electrical resistivity, thermoelectric power (TEP) measurements, molecular dynamics (MD) simulations, and ab-initio calculations. The results of MD simulations and extended X-ray absorption fine structure analysis indicate that atomic order of $\mathrm{Cu}-\mathrm{Zr}$ MGs and can be described in terms of interpenetrating icosahedral-like clusters involving five-fold symmetry. MD configurations were used as an input for calculations of theoretical electronic density of states (DOS) functions which exhibits good agreement with the experimental X-ray absorption near-edge spectra. We found no indication of minimum of DOS at Fermi energy predicted by Mott's nearly free electron (NFE) model for glass-forming alloys. The theoretical DOS was subsequently used to test Mott's model describing the temperature variation of electrical resistivity and thermoelectric power of transition metal-based MGs. We demonstrate that the measured temperature variations of electrical resistivity and TEP remain in a contradiction with this model. On the other hand, the experimental temperature dependence of electrical resistivity can be explained by incipient localization of conduction electrons. It is shown that weak localization model works up to relatively high temperatures when localization is destroyed by phonons. Our results indicate that electron transport properties of $\mathrm{Cu}-\mathrm{Zr}$ MGs are dominated by localization effects rather than by electronic structure. We suggest that NFE model fails to explain a relatively high glass-forming ability of binary $\mathrm{Cu}-\mathrm{Zr}$ alloys. (C) 2014 AIP Publishing LLC. [http://dx.doi.org/10.1063/1.4879903]
\end{abstract}

\section{INTRODUCTION}

Phase stability of metallic glasses (MGs) ${ }^{1}$ is usually considered in terms of atomic size difference and attractive interatomic interactions between unlike species giving rise to negative enthalpy of mixing. ${ }^{2}$ The enhanced stability of glassforming metallic liquids leading to high glass-forming ability (GFA) results from complex interrelation between atomic structure (high packing efficiency, ${ }^{3}$ local symmetry, ${ }^{4}$ atomiclevel stress ${ }^{5}$ ) and electronic properties (bond characteristics, ${ }^{6}$ electronic density of states (DOS) near Fermi energy, ${ }^{7}$ valence electron concentration ${ }^{8}$ ). As MGs lack translational symmetry, the atoms are ordered on a length scale ranging from nearestneighbors distances (short range order-SRO) up to about 1-2 nm (medium range order-MRO). SRO is achieved by efficient packing within a single cluster while the spacial arrangement of interpenetrating clusters corresponds to MRO. ${ }^{9}$ Among numerous glass-forming metallic alloys, a prominent group consists of transition metal (TM)-based MGs. Binary $\mathrm{Cu}-\mathrm{Zr}$ system ${ }^{10-16}$ is a prototype of many bulk metallic glasses ${ }^{17}$ exhibiting excellent mechanical properties. ${ }^{2}$

Atomic order of MGs is typically determined either from diffraction of x-rays, electrons or neutrons or by direct imaging by high resolution electron microscopy. X-ray absorption fine structure (XAFS) ${ }^{18}$ is an alternative method

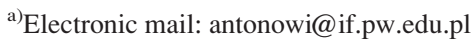

of investigating of atomic arrangement in amorphous metals. While XAFS is not sensitive to long-range order it provides accurate information on SRO-coordination numbers, bonding distances, and local symmetry around absorbing atomic species. During absorption of an X-ray photon, an electron is promoted from a deep core level to an unoccupied state above Fermi energy. For this reason, XAFS brings information not only on atomic but also on electronic band structure of material. Those two kinds of information are important for understanding of fundamental mechanism of glass formation in metallic alloy systems.

Electron transport properties of MGs (electrical resistivity and thermoelectric power (TEP)) are determined by nonperiodic atomic arrangement, electron-phonon scattering and by electronic band structure. ${ }^{19}$ Early approaches of electron transport in amorphous metals treat them as nearly freeelectron (NFE) gas. In Ziman theory, ${ }^{20,21}$ the value of resistivity and thermal coefficient of resistivity (TCR) are determined by the relation between the position of a peak $Q_{p}$ in the structure factor $S(Q)$ and Fermi momentum $k_{F}$. Mott ${ }^{22}$ explained the reason for the possession of a relatively large resistivity in liquid TMs in terms of so called $s-d$ scattering model assuming that only $s p$ electrons are responsible for electron conductivity. Inelastic electron-phonon interactions determine the temperature dependence of the electrical resistivity in amorphous alloys but its contribution becomes less important as the residual resistivity (resistivity at absolute zero) increases. The residual 
resistivity arises from the elastic scattering of conduction electrons due to random distributions of ions. Anderson ${ }^{23}$ showed that all electrons within the band cannot form the Bloch wave but tend to localize in real space due to repeated elastic scattering events of conduction electrons on randomly distributed ions. As inelastic scattering does not occur at $0 \mathrm{~K}$, Anderson weak localization is likely to occur in systems possessing high residual resistivity. At $\mathrm{T}>0 \mathrm{~K}$, electrons are scattered inelastically by phonons so their phase coherence is gradually destroyed during the successive scattering events leading to delocalization. The above mechanism yields a negative TCR in the temperature dependence of the electrical resistivity. The mechanisms of electron localization in amorphous metallic alloys have been discussed in detail in review papers by Howson $^{24}$ and Dugdale. ${ }^{25}$ As pointed out by Altshuler and Aronov, ${ }^{26}$ localization of conduction electrons weakens the screening effect and enhances electron-electron Coulomb interactions affecting the temperature dependence of electrical conductivity $\sigma(T)$. Enhanced electron-electron interaction and weak localization effect are beyond the NFE model thus the validity of the NFE approach can be verified from temperature measurements of electron transport properties.

Nagel and Tauc ${ }^{7}$ used the NFE approach to introduce a criterion for formation of MGs from liquid alloys. According to them, when the Fermi sphere just touches the quasiBrillouin zone, i.e., $Q_{p}=2 k_{F}$ condition is fulfilled an isotropic "pseudogap" arises at Fermi energy in the electronic DOS. ${ }^{27}$ The "pseudogap" leads to lowering of kinetic energy of the conduction electrons in the liquid (or glassy) phase relative to crystalline state. Nagel and Tauc argue that amorphous alloy for which condition $Q_{p}=2 k_{F}$ is satisfied will posses higher stability against crystallization than systems where $Q_{p} \neq 2 k_{F}$. The validity of the above criterion has been verified for various glass forming metallic alloys by relating the GFA with equality of $k_{F}$ and $Q_{p} \cdot{ }^{28-31}$ While $Q_{p}=2 k_{F}$ criterion was generally accepted for simple metal alloys, its applicability for transition metal alloys involving $d$-electrons is disputable. ${ }^{28,32}$

In this study, we present a consistent picture of atomic and electronic structures of $\mathrm{Cu}-\mathrm{Zr}$ binary amorphous alloys. We apply XAFS results to validate the results of molecular dynamics (MD) simulations and ab-initio calculations of the band structure. Electrical resistivity and TEP measurements were used to verify the degree of applicability of NFE approach to $\mathrm{Cu}-\mathrm{Zr}$ MGs. It is shown that amorphous phase in $\mathrm{Cu}-\mathrm{Zr}$ system is not stabilized by favorable electronic energy contribution.

\section{EXPERIMENTAL}

Ingots of investigated alloys were prepared from highpurity elements (99.999\%) using arc-melting applying multiple remelting to ensure homogeneity. Glassy samples were prepared by rapid quenching using single-roller melt spinning setup operating under argon atmosphere. A fully amorphous character of the samples was confirmed by x-ray diffraction measurements. As-quenched samples were in form of $\sim 30 \mu \mathrm{m}$ thick and $\sim 2 \mathrm{~mm}$ wide ribbons.

XAFS experiments were carried out at $\mathrm{X} 1$ beamline of HASYLAB. The absorption spectra of $\mathrm{Cu}_{35} \mathrm{Zr}_{65}$ and $\mathrm{Cu}_{65} \mathrm{Zr}_{35}$ alloys were measured for both $\mathrm{Cu}$ and $\mathrm{Zr} \mathrm{K}$-edge in transmission mode using ionisation chambers. The samples in form of melt-spun ribbons were cooled down to liquid nitrogen temperature to minimize thermal disorder. XAFS data reduction was performed using IFEFFIT software package. ${ }^{33}$ FEFF8 (Ref. 34) code was used for ab-initio calculations of theoretical extended X-ray absorption fine structure (EXAFS) spectra and electronic DOS functions. The details of the EXAFS experiment and theoretical EXAFS signal calculation are described elsewhere. ${ }^{35}$

Large scale MD simulations were carried out using embedded atom model (EAM) potential that has given reliable results concerning the microstructure of the glassy systems. Periodic boundary conditions in three directions were applied for a system in form of a cubic box having edge length of $10.85 \mathrm{~nm}$ and consisting of 78.608 atoms. The system was initially equilibrated at $2000 \mathrm{~K}$ temperature for $30 \mathrm{ps}$ and subsequently was cooled down to room temperature at a cooling rate of $2 \mathrm{~K} / \mathrm{ps}$. The final configurations were equilibrated at room temperature for $150 \mathrm{ps}$. Given that no significant structural differences were observed for system quenched at $0.1 \mathrm{~K} / \mathrm{ps}$, we assumed that the equilibrium configuration is not considerably affected by high cooling rate imposed by the time limitations inherent to the MD simulations.

Electrical resistivity of the as-quenched ribbons was measured using DC four-probe method applying current below $10 \mathrm{~mA}$. The resistivity measurements in temperature range of $20-300 \mathrm{~K}$ were carried out using the closed cycle refrigerator providing temperature stability $\pm 0.02 \mathrm{~K}$, while the data above room temperature were acquired using high temperature setup with accuracy of $0.1 \%$. TEP was measured applying differential method using $\sim 20 \mathrm{~K}$ temperature gradient. Thermal stability of the glassy samples was investigated by means of differential scanning calorimetry (DSC) (DSC Q200 TA Instruments). The glass transition $T_{g}$ and crystallization $T_{x}$ temperatures were determined in continuous heating conditions $(10 \mathrm{~K} / \mathrm{min})$ using pure nitrogen purge flow of $50 \mathrm{ml} / \mathrm{min}$.

\section{RESULTS AND DISCUSSION}

Figure 1 shows the DSC traces of the investigated glassy samples. In all cases, the samples exhibit glass transition

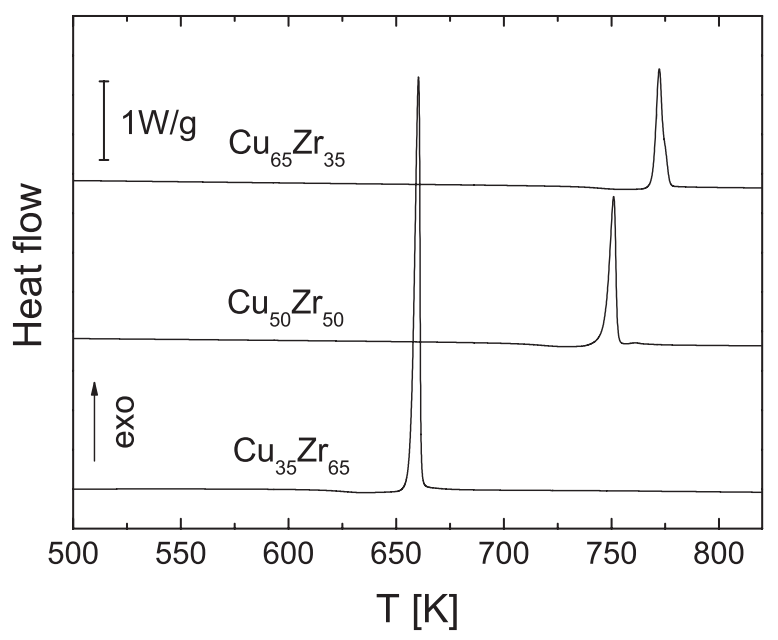

FIG. 1. DSC traces of investigated $\mathrm{Cu}-\mathrm{Zr}$ glassy alloys. Curves are shifted vertically of clarity. 
TABLE I. Glass transition temperature $T_{g}$, crystallization temperature $T_{x}$, supercooled liquid region $\Delta T_{x}$, liquidus temperature $T_{l}$ (taken from Ref. 36), and reduced glass transition temperature $T_{r g}$ of $\mathrm{Cu}-\mathrm{Zr}$ alloys studied in this work.

\begin{tabular}{lccccc}
\hline \hline Alloy & $T_{g}(\mathrm{~K})$ & $T_{x}(\mathrm{~K})$ & $\Delta T_{x}(\mathrm{~K})$ & $T_{l}(\mathrm{~K})$ & $T_{r g}$ \\
\hline $\mathrm{Cu}_{35} \mathrm{Zr}_{65}$ & 627 & 654 & 27 & 1273 & 0.49 \\
$\mathrm{Cu}_{50} \mathrm{Zr}_{50}$ & 718 & 741 & 23 & 1208 & 0.59 \\
$\mathrm{Cu}_{65} \mathrm{Zr}_{35}$ & 745 & 766 & 21 & 1248 & 0.60 \\
\hline \hline
\end{tabular}

followed by crystallization of the supercooled liquid manifested by a sharp exothermic peak. Thermal stability parameters $\left(T_{g}, T_{x}\right.$, supercooled liquid region width $\Delta T_{x}=T_{x}-T_{g}$, $T_{l}$, and reduced glass transition temperature $\left.T_{r g}=T_{g} / T_{l}\right)$ are summarized in Table I. The comparison shows that as the $\mathrm{Cu}$ content increases $T_{g}, T_{x}$ and $T_{r g}=T_{g} / T_{l}$ increase while $\Delta T_{x}$ is reduced.

In the following part of this section, we address the question of significant electron contribution to the thermal stability and glass-forming ability of Cu-Zr MGs. Namely, we employ MD simulations to obtain realistic structural models of the investigated amorphous systems and subsequently use the MD configurations to calculate the DOS functions. To make sure that the computer-simulated configurations are representative for the actual atomic structure of the glassy alloys, we used EXAFS method as a tool allowing direct and accurate comparison between theory and experiment. As the signal measured in the EXAFS experiment is an average of the single EXAFS functions of all the absorbing atoms present in the irradiated volume, the theoretical EXAFS function was calculated by averaging over 600 random atoms randomly picked from the MD-simulation box. The comparison of the experimental and the theoretical $\mathrm{Cu}$ and $\mathrm{Zr}$ K-edge EXAFS functions for $\mathrm{Cu}_{35} \mathrm{Zr}_{65}$ and $\mathrm{Cu}_{65} \mathrm{Zr}_{35}$ metallic glass is demonstrated in Fig. 2. Some important points should be noted here. First, our approach does not

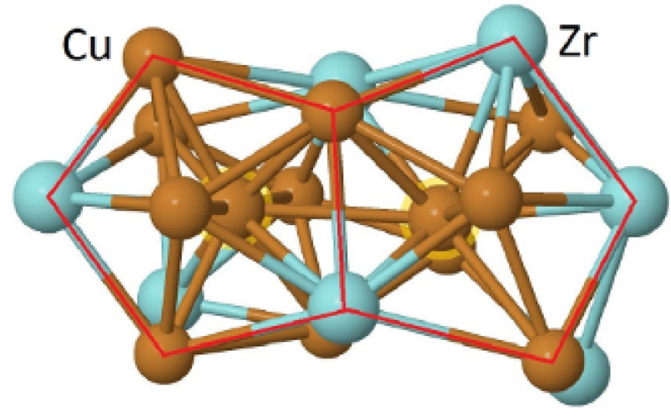

FIG. 3. 20-atom supercluster consisting of two overlapping $\mathrm{Cu}$-centered icosahedral-like clusters. The central atoms of the clusters are highlighted and the five-fold symmetry elements are indicated.

involve any kind of fitting and is based essentially on ab-initio calculations with no free parameters. Second, as EXAFS is extremely sensitive to short-rage order, even small structural difference between the real and computer-simulated systems is clearly visible. Due to ultra-high cooling rate which is unavoidable in MD simulations, the simulated system is far less relaxed than the melt-quenched glassy alloy. Different relaxation state of the MD configuration may slightly affect its short-range order characteristics. One should also keep in mind that while EXAFS experiments were carried out at liquid nitrogen temperature $(80 \mathrm{~K})$ and thus the structure of the measured samples involves some thermal disorder, the MD configuration is completely static. Finally, the ab-initio calculations performed with $F E F F 8$ code involve set of approximations $^{34}$ which to some extend affect the theoretical EXAFS. Considering the above issues, we conclude that the comparison demonstrated in Fig. 2 is satisfactory and that our MD simulations reproduce the essential short-range order features of $\mathrm{Cu}-\mathrm{Zr}$ glassy alloys. Fig. 3 shows the typical structural motif of the MD configuration-a "supecluster" 35,37 consisting of two interpenetrating icosahedral-like clusters. In our
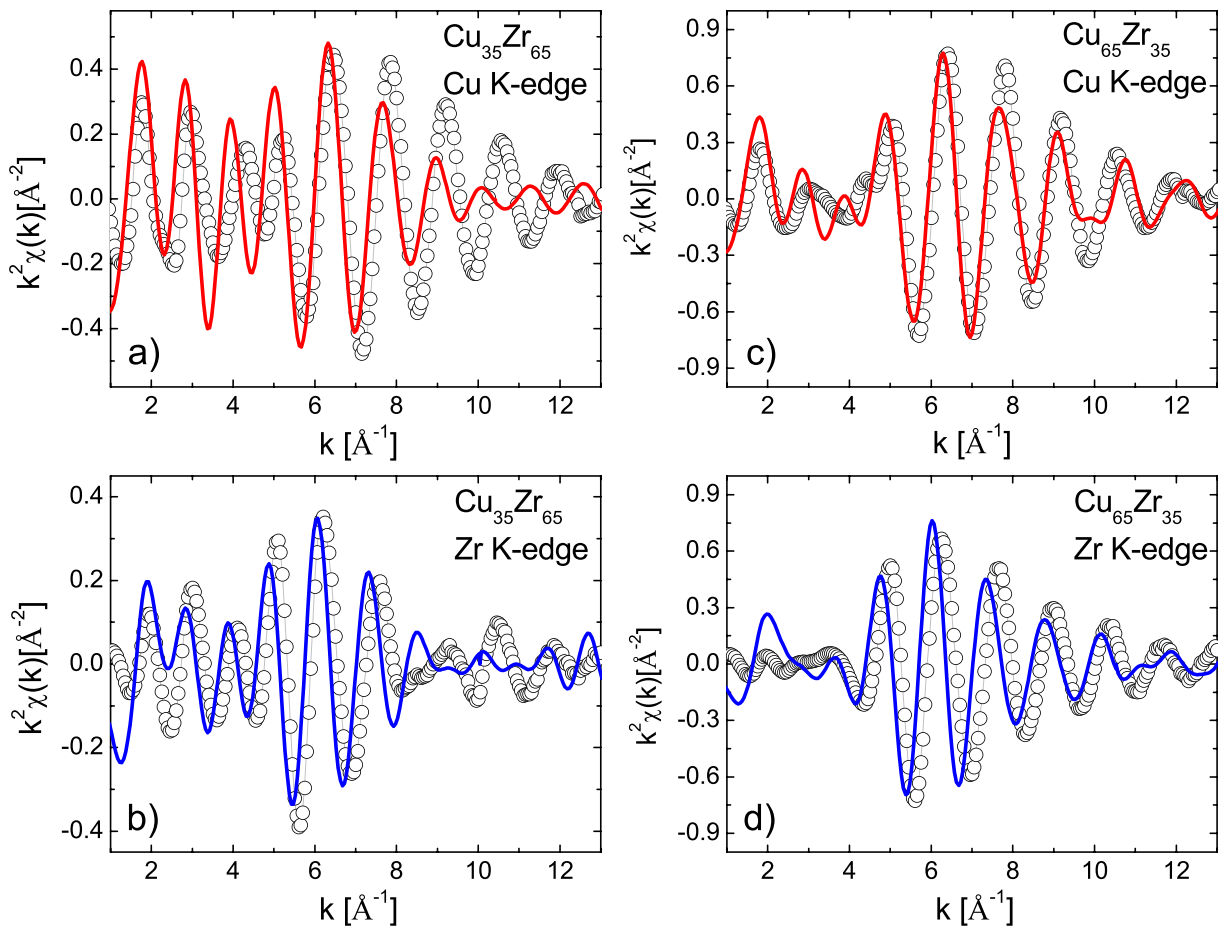

FIG. 2. Experimental $\mathrm{Cu}$ and $\mathrm{Zr}$ K-edge $k^{2}$-weighted EXAFS function for $\mathrm{Cu}_{35} \mathrm{Zr}_{65}$ ((a) and (b)) $\mathrm{Cu}_{65} \mathrm{Zr}_{35}$ ((c) and (d)) metallic glasses (symbols) and theoretical EXAFS (line) obtained by averaging over 600 atoms picked randomly from the MD configurations. 

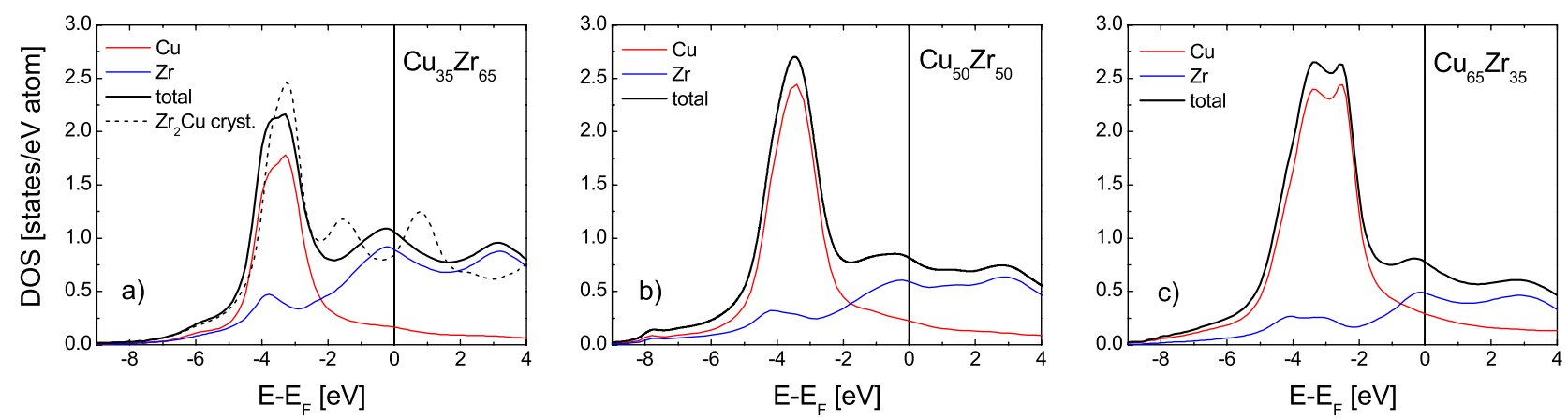

FIG. 4. Calculated densities of states of $\mathrm{Cu}_{35} \mathrm{Zr}_{65}$ (a), $\mathrm{Cu}_{50} \mathrm{Zr}_{50}$ (b), and $\mathrm{Cu}_{65} \mathrm{Zr}_{35}$ (c) glasses. The contributions from $\mathrm{Cu}$ and $\mathrm{Zr}$ atoms are indicated. The dashed line represents the DOS function of $\mathrm{Zr}_{2} \mathrm{Cu}$ intermetallic compound. The vertical line at $E-E_{F}=0$ is drawn to guide the eye.

analysis of superclusters' population, we have considered only nearly perfectly icosahedral clusters. We have found that fractions of icosahedral clusters (in atoms with respect to the total number of systems' atoms) are 15\%, 18\%, and 32\% for $\mathrm{Cu}_{35} \mathrm{Zr}_{65}, \mathrm{Cu}_{50} \mathrm{Zr}_{50}$, and $\mathrm{Cu}_{65} \mathrm{Zr}_{35}$, respectively.

MD simulation data were used as an input for calculations of the DOS function for $\mathrm{Cu}-\mathrm{Zr}$ amorphous alloys. The calculations were performed with FEFF8 software for clusters of about 60 atoms (about $1.2 \mathrm{~nm}$ size) extracted from the MD configuration and the final DOS functions were obtained by averaging over about 5 clusters atoms involving 300 atoms overall. We found that including larger number atoms does not significantly affect the resulting DOS. Figure 4 shows the theoretical DOS functions of $\mathrm{Cu}_{35} \mathrm{Zr}_{65}, \mathrm{Cu}_{50} \mathrm{Zr}_{50}$, and $\mathrm{Cu}_{65} \mathrm{Zr}_{35}$ amorphous alloys as well as crystalline $\mathrm{Zr}_{2} \mathrm{Cu}$ intermetallic compound. All systems exhibit a pronounced maximum located around $3.5 \mathrm{eV}$ below $E_{F}$ which is attributed to copper $d$-states while the density of states in the vicinity of Fermi level and above are mainly due to the $d$-states of zirconium. Apparently none of the glassy systems exhibits a minimum of density of states located at Fermi energy. On the contrary, in all three cases a maximum of DOS function is observed slightly below $E_{F}$. Interestingly, a noticeable minimum at $E_{F}$ is visible for $\mathrm{Zr}_{2} \mathrm{Cu}$ compound-a crystalline polymorph of $\mathrm{Cu}_{35} \mathrm{Zr}_{65}$ glassy alloy. The above observations indicate that electronic structure of $\mathrm{Cu}_{35} \mathrm{Zr}_{65}$ metallic glass is fundamentally different from that of relevant crystalline compound. It should be mentioned that very similar results on electronic structure of $\mathrm{Zr}-\mathrm{Cu}$ MGs were reported previously. ${ }^{38-41}$

In order to verify the theoretical DOS functions, we employed the near-edge part of the XAFS spectra (XANES-X-ray absorption near-edge structure). As $\mathrm{K}$ absorption edge corresponds to electron transition from $1 s$ to an unoccupied $2 p$ states the XANES spectrum pictures partial density of $p$-states above Fermi energy. Figure 5 shows of the experimental $\mathrm{Cu} \mathrm{K}$-edge XANES together with calculated $p$ DOS of $\mathrm{Cu}_{35} \mathrm{Zr}_{65}$ and $\mathrm{Cu}_{65} \mathrm{Zr}_{35}$ MGs. It is clear that the theoretically evaluated $p$-DOS perfectly reproduces all details of the XANES-both the pre-edge feature as well as oscillations of the absorption coefficient. Given that DOS calculations were carried out utilizing a self-consistent code $(F E F F)$, we conclude that the total density of states consisting of partial $s$, $p$, and $d$-DOS functions are realistic and accurately describe the electronic structure of investigated amorphous systems.

The lack of DOS minimum at the Fermi level indicates that the condition $Q_{p}=2 k_{F}$ is not satisfied in the case of binary $\mathrm{Cu}-\mathrm{Zr}$ amorphous alloys. This can be understood given the fact that the electron structure of TM-based MGs is dominated by the $d$ electron states which undermines validity of nearly free electron approximation. Incipient localization of $d$ electrons affects the electron transport properties of the glassy alloy and may be detected by careful analysis of temperature dependence of electrical resistivity and thermoelectric power.

According to Mott NFE model electrical resistivity $\rho$ of a TM-based amorphous metallic alloy scales with the value density of the $d$-states at Fermi energy $N_{d}\left(E_{F}\right)^{19}$

$$
\rho \propto N_{d}\left(E_{F}\right)
$$

and TEP (Seebeck coefficient) is function of both $N_{d}\left(E_{F}\right)$ and its slope $\left(\partial N_{d} / \partial E\right)_{E_{F}}$

$$
S=\frac{\pi^{2} k_{B}^{2}}{3 e} T \frac{1}{N_{d}\left(E_{F}\right)}\left(\frac{\partial N_{d}}{\partial E}\right)_{E_{F}},
$$

where $k_{B}$ is Boltzmann constant, $e$ is elementary charge, and $T$ is temperature. Table II presents experimental values of room temperature resistivity, TCR, and Seebeck coefficient together with calculated values of $N_{d}\left(E_{F}\right),\left(\partial N_{d} / \partial E\right)_{E_{F}}$ and Seebeck coefficient. According to classification proposed by
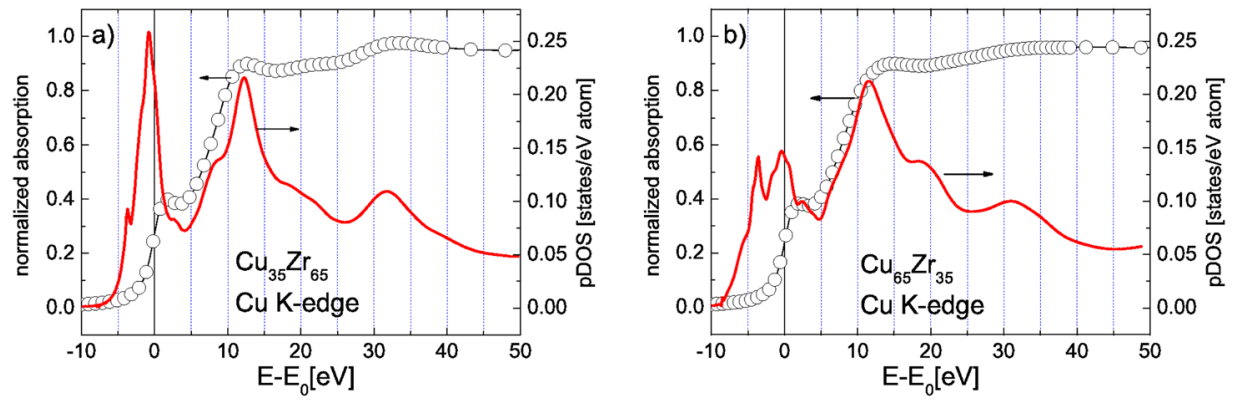

FIG. 5. Experimental XANES Cu Kedge spectra for $\mathrm{Cu}_{35} \mathrm{Zr}_{65}$ (a) and $\mathrm{Cu}_{65} \mathrm{Zr}_{35}$ (b) alloys (symbols) and theoretical densities of $\mathrm{Cu} p$-states (line). 
TABLE II. Experiment values of room temperature electrical resistivity $\rho_{300 \mathrm{~K}}$, thermal coefficient of electrical resistivity at (TCR), Seebeck coefficient $S_{300 K}$, theoretical density of $d$-states at Fermi energy $N_{d}\left(E_{F}\right)$, derivative of density of $d$-states at Fermi energy $\left(\partial N_{d} / \partial E\right)_{E_{F}}$, and calculated Seebeck coefficient $S_{300 \mathrm{~K}}^{\text {calc }}$.

\begin{tabular}{|c|c|c|c|c|c|c|}
\hline Composition & $\rho_{300 \mathrm{~K}}(\mu \Omega \mathrm{cm})$ & $\mathrm{TCR}\left(\mathrm{K}^{-1}\right)$ & $S_{300 \mathrm{~K}}(\mu \mathrm{V} / \mathrm{K})$ & $N_{d}\left(E_{F}\right)\left(\mathrm{eV}^{-1}\right)$ & $\left(\partial N_{d} / \partial E\right)_{E_{F}}\left(\mathrm{eV}^{-2}\right)$ & $S_{300 \mathrm{~K}}^{c a l c}(\mu \mathrm{V} / \mathrm{K})$ \\
\hline $\mathrm{Cu}_{35} \mathrm{Zr}_{65}$ & $174 \pm 5$ & $-1.4 \times 10^{-4}$ & $2.9 \pm 0.1$ & 0.82 & 0.22 & 2.5 \\
\hline $\mathrm{Cu}_{50} \mathrm{Zr}_{50}$ & $174 \pm 5$ & $-0.9 \times 10^{-4}$ & $2.5 \pm 0.1$ & 0.62 & 0.20 & 1.8 \\
\hline $\mathrm{Cu}_{65} \mathrm{Zr}_{35}$ & $190 \pm 5$ & $-0.7 \times 10^{-4}$ & $2.3 \pm 0.1$ & 0.60 & 0.25 & 2.4 \\
\hline
\end{tabular}

Mizutani, ${ }^{42} \mathrm{Cu}-\mathrm{Zr}$ MGs are high-resistivity alloys with negative TCR. Thermoelectric power is negative for all composition and fairly small in magnitude. ${ }^{19}$ The theoretical values of Seebeck coefficient calculated using Eq. (2) are close to experimental ones. However, it should be noted that Eq. (2) involves slope of density of $d$-states at Fermi energy and $d$-DOS exhibits a maximum located very near $E_{F}$. For this reason, $\left(\partial N_{d} / \partial E\right)_{E_{F}}$ and consequently $S$ are very sensitive to small variations of $E_{F}$ resulting from ab-initio calculations which makes uncertainty if $S$ rather large and difficult to quantify. From the data shown in Table II, it can be seen that while $N_{d}\left(E_{F}\right)$ decreases with decreasing $\mathrm{Zr} / \mathrm{Cu}$ ratio the value of resistivity exhibits an opposite trend. This observation indicates that relation $\rho \propto N_{d}\left(E_{F}\right)$ does not hold and thus the applicability of NFE model is strongly limited in case of $\mathrm{Cu}-\mathrm{Zr}$ amorphous alloys.

To get better insight into electron transport properties of $\mathrm{Cu}-\mathrm{Zr}$ MGs, we analysed temperature dependencies of electrical resistivity and thermoelectric power which carry important information of quantum effects such as localization and interaction in amorphous alloys. The quantum corrections to classical weak-scattering (or nearly free electrons) models ${ }^{20}$ are particularly important for high-resistivity alloys with negative TCR. In case of strong electron scattering, the electron mean-free path becomes comparable with the interatomic distance the quantum corrections can dominate

a)
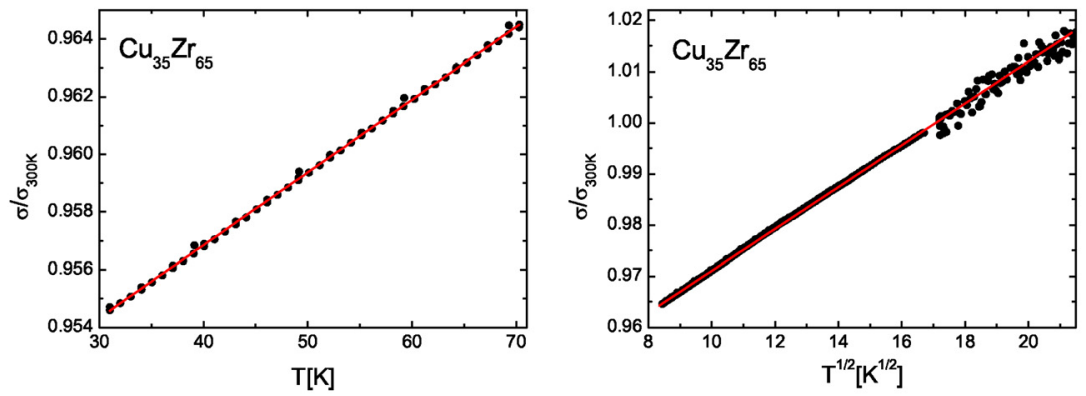

b)
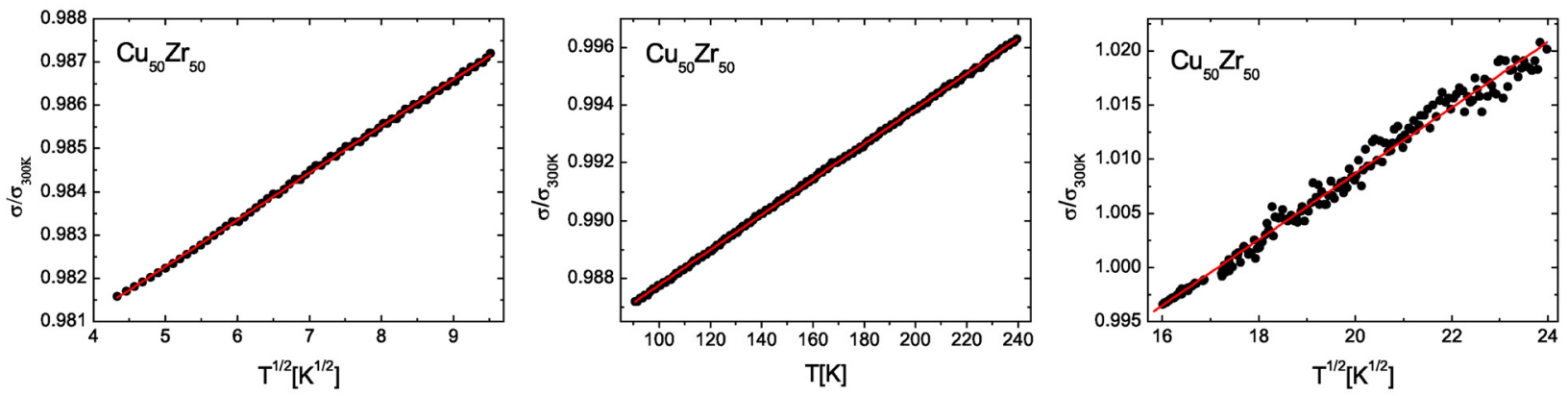

c)
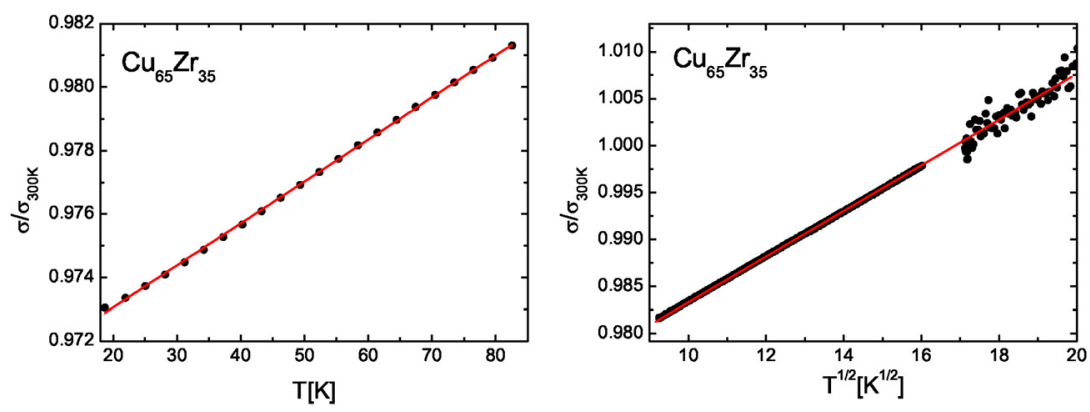

FIG. 6. Normalized electrical conductivity $\sigma(T) / \sigma(300 \mathrm{~K})$ plotted as a function of temperature and square root of temperature for $\mathrm{Cu}_{35} \mathrm{Zr}_{65}(\mathrm{a}), \mathrm{Cu}_{50} \mathrm{Zr}_{50}$ (b), and $\mathrm{Cu}_{65} \mathrm{Zr}_{35}$ (c). The straight lines are linear fits for different temperature ranges. 
TABLE III. $T$ and $\sqrt{T}$ proportionality ranges of electrical conductivity determined from the experimental $\sigma(T)$ functions.

\begin{tabular}{lcc}
\hline \hline Composition & $\sigma \sim T$ range & $\sigma \sim \sqrt{T}$ range \\
\hline $\mathrm{Cu}_{35} \mathrm{Zr}_{65}$ & $30 \mathrm{~K}-70 \mathrm{~K}$ & $70 \mathrm{~K}-460 \mathrm{~K}$ \\
$\mathrm{Cu}_{50} \mathrm{Zr}_{50}$ & $90 \mathrm{~K}-240 \mathrm{~K}$ & $20 \mathrm{~K}-90 \mathrm{~K} ; 240 \mathrm{~K}-580 \mathrm{~K}$ \\
$\mathrm{Cu}_{65} \mathrm{Zr}_{35}$ & $20 \mathrm{~K}-90 \mathrm{~K}$ & $90 \mathrm{~K}-400 \mathrm{~K}$ \\
\hline \hline
\end{tabular}

electron transport properties. As pointed out by Bergmann ${ }^{43}$ after repeated elastic scattering events, the conduction electrons tend to return to their primary scattering point due to phase coherence between scattered partial waves. This phenomenon leads to incipient localization of the conduction electrons and is often referred to as " $2 k_{F}$ scattering." By using scaling approach to localization and some simplifications, ${ }^{24}$ the correction to Boltzmann electrical conductivity $\sigma$ ( $\sigma=1 / \rho$, where $\rho$ is electrical resistivity) obeys $T$-dependence below $\theta_{D} / 3$ and $\sqrt{T}$-dependence above $\theta_{D} / 3\left(\theta_{D}\right.$ is Debye temperature). At low temperatures, the localization process can enhance Coulombic electron-electron interactions which lead to $\sqrt{T}$-dependence of $\sigma{ }^{26} \mathrm{~A}$ characteristic feature of high-resistivity amorphous alloys is the existence of $\sqrt{T} \rightarrow T \rightarrow \sqrt{T}$ transitions sequence of $\sigma(T)$. Figure 6 shows the normalized electrical conductivity $\sigma(T) / \sigma(300 \mathrm{~K})$ plotted as a function of temperature and square root of temperature for investigated Cu-Zr MGs. All alloys exhibit $T \rightarrow \sqrt{T}$ transition in the investigated temperature range and in addition $\mathrm{Cu}_{50} \mathrm{Zr}_{50}$ exhibits a low temperature $\sqrt{T} \rightarrow T$ transition. Different temperature proportionality ranges of $\sigma(T)$ are summarized in Table III. Lack of $\sqrt{T} \rightarrow T$ transition for $\mathrm{Cu}_{35} \mathrm{Zr}_{65}$ and $\mathrm{Cu}_{65} \mathrm{Zr}_{35}$ may be explained by the fact that electron-electron interaction term is often significant below $20 \mathrm{~K}$ (Ref. 19) which is beyond the temperature range available in the experimental setup used in our study. Judging from the electrical conductivity data, it is clear that electron localization effect is significant in case of $\mathrm{Cu}-\mathrm{Zr}$ amorphous alloys and the localization persists up to relatively high temperatures: $170 \mathrm{~K}, 140 \mathrm{~K}$, and $345 \mathrm{~K}$ below $T_{g}$ in the cases of $\mathrm{Cu}_{35} \mathrm{Zr}_{65}, \mathrm{Cu}_{50} \mathrm{Zr}_{50}$, and $\mathrm{Cu}_{65} \mathrm{Zr}_{35}$, respectively. The above results indicate that resistivity of $\mathrm{Cu}-\mathrm{Zr}$ MGs cannot be understood within the nearly free electron approximation and their topologically disordered atomic structure strongly contributes to electron transport.

\section{CONCLUSIONS}

In this work, we have studied atomic and electronic structure as well as electron transport properties of three binary $\mathrm{Cu}-\mathrm{Zr}$ rapidly quenched amorphous alloys $\left(\mathrm{Cu}_{35} \mathrm{Zr}_{65}\right.$, $\mathrm{Cu}_{50} \mathrm{Zr}_{50}$, and $\mathrm{Cu}_{65} \mathrm{Zr}_{35}$ ) employing XAFS spectroscopy, MD simulations, ab-initio calculations, and electron transport measurements. By using various experimental and computational methods, we present a consistent description of atomic and electronic structures of $\mathrm{Cu}-\mathrm{Zr}$ MGs. The atomic structure of $\mathrm{Cu}-\mathrm{Zr}$ MGs consists of interpenetrating icosahedral-like clusters involving five-fold symmetry. Calculated density of states of none of investigated alloys exhibits a minimum located at Fermi energy ("pseudogap") as predicted by NFE model. From the detailed analysis of electron transport properties, we demonstrate that NFE approximation is not sufficient to describe electrical resistivity of investigated systems because of quantum corrections to conductivity originating from structural disorder of $\mathrm{Cu}-\mathrm{Zr}$ MGs. In conclusion, we suggest that relatively good glass forming ability in $\mathrm{Cu}-\mathrm{Zr}$ system cannot be readily explained by lowering of system energy due to favorable electronic energy contribution.

${ }^{1}$ A. L. Greer, Science 267, 1947-1953 (1995).
${ }^{2}$ A. Inoue, Acta Mater. 48, 279-306 (2000).
${ }^{3}$ D. B. Miracle, Nature Mater. 3, 697-702 (2004).

${ }^{4}$ H. W. Sheng, W. K. Luo, F. M. Alamgirand, J. M. Bai, and E. Ma, Nature 439, 419-425 (2006).

${ }^{5}$ T. Egami and Y. Waseda, J. Non-Cryst. Solids 64, 113-134 (1984).

${ }^{6}$ Ch. E. Lekka and G. A. Evangelakis, Scr. Mater. 61(10), 974-977 (2009).

${ }^{7}$ S. R. Nagel and J. Tauc, Phys. Rev. Lett. 35, 380-383 (1975).

${ }^{8}$ Q. Jiang, B. Q. Chi, and J. C. Li, Appl. Phys. Lett. 82, 2984-2986 (2003).

${ }^{9}$ A. R. Yavari, Nature 439, 405-406 (2006).

${ }^{10}$ R. Ray, B. C. Giessen, and N. J. Grant, Scr. Metall. 2(6), 357-359 (1968).

${ }^{11}$ A. Inoue and W. Zhang, Mater. Trans., JIM 45, 584-587 (2004).

${ }^{12}$ D. Xu, B. Lohwongwatana, G. Duan, W. L. Johnson, and C. Garland, Acta Mater. 52(9), 2621-2624 (2004).

${ }^{13}$ J. Saida, T. Sanada, S. Sato, M. Imafuku, E. Matsubara, and A. Inoue, Mater. Trans. 48(7), 1703-1707 (2007).

${ }^{14}$ D. V. Louzguine-Luzgin, K. Georgarakis, A. R. Yavari, G. Vaughan, G. Xie, and A. Inoue, J. Mater. Res. 24(1), 274-278 (2009).

${ }^{15}$ N. Mattern, P. Jívári, I. Kaban, S. Gruner, A. Elsner, V. Kokotin, H. Franz, B. Beuneu, and J. Eckert, J. Alloys Compd. 485, 163-169 (2009).

${ }^{16}$ K. Georgarakis, A. R. Yavari, D. V. Louzguine-Luzgin, J. Antonowicz, M. Stoica, Y. Li, M. Satta, A. LeMoulec, G. Vaughan, and A. Inoue, Appl. Phys. Lett. 94, 191912 (2009).

${ }^{17}$ A. Peker and W. L. Johnson, Appl. Phys. Lett. 63, 2342 (1993).

${ }^{18}$ J. J. Rehr and R. C. Albers, Rev. Mod. Phys. 72, 621-654 (2000).

${ }^{19}$ U. Mizutani, Introduction to the Electron Theory of Metals (Cambridge University Press, 2001).

${ }^{20}$ J. M. Ziman, Philos. Mag. 6, 1013 (1961).

${ }^{21}$ P. J. Cote and L. V. Meisel, Phys. Rev. B 20, 3030-3035 (1979).

${ }^{22}$ N. F. Mott, Philos. Mag. 19(160), 835-852 (1969).

${ }^{23}$ P. W. Anderson, Phys. Rev. 109, 1492-1505 (1958).

${ }^{24}$ M. A. Howson and B. L. Gallagher, Phys. Rep. 170, 265-324 (1988).

${ }^{25}$ J. S. Dugdale, Contemp. Phys. 28, 547-572 (1987).

${ }^{26}$ B. L. Altshuler and A. G. Aronov, Electron-Electron Interactions in Disordered Systems (Elsevier Science Publisher, 1985).

${ }^{27}$ P. Haussler, Z. Phys. B: Condens. Matter 53(1), 15-26 (1983).

${ }^{28}$ U. Mizutani, K. T. Hartwig, T. B. Massalski, and R. W. Hopper, Phys. Rev. Lett. 41, 661-664 (1978).

${ }^{29}$ K. H. J. Bushow, J. Phys. F: Met. Phys. 13, 563-571 (1983).

${ }^{30}$ S. Hosokawa, H. Sato, N. Happo, K. Mimura, Y. Tezuka, T. Ichitsubo, E. Matsubara, and N. Nishiyama, Acta Mater. 55, 3413-3419 (2007).

${ }^{31}$ H. B. Yu, W. H. Wang, and H. Y. Bai, Appl. Phys. Lett. 96, 081902 (2010).

${ }^{32} \mathrm{R}$. Hasegawa and K. Tanaka, MRS Proceedings 58, 53 (1985).

${ }^{33}$ B. Ravel and M. Newville, J. Synchrotron Radiat. 12, 537-541 (2005).

${ }^{34}$ A. L. Ankudinov, B. Ravel, J. J. Rehr, and S. D. Conradson, Phys. Rev. B 58, 7565-7576 (1998).

${ }^{35}$ J. Antonowicz, A. Pietnoczka, T. Drobiazg, G. A. Almyras, D. G. Papageorgiou, and G. A. Evangelakis, Philos. Mag. A 92(15), 1865-1875 (2012).

${ }^{36}$ P. R. Subramanian, Phase Diagrams of Binary Copper Alloys (ASM International, 1993).

${ }^{37}$ G. A. Almyras, Ch. E. Lekka, N. Mattern, and G. A. Evangelakis, Scr. Mater. 62, 33-36 (2010).

${ }^{38}$ M. Hasegawa, H. Sato, T. Takeuchi, K. Soda, and U. Mizutani, J. Alloys Compd. 483, 638-641 (2009).

${ }^{39}$ S. Frota-Pessôa, Phys. Rev. B 28, 3753-3758 (1983).

${ }^{40}$ D. Nguyen Manh, D. Mayou, F. Cyrot-Lackmann, and A. Pasturel, J. Phys. F: Met. Phys. 17(6), 1309-1321 (1987).

${ }^{41}$ Y. Takahara and N. Narita, Mater. Trans. 45, 1172-1176 (2004).

${ }^{42}$ U. Mizutani, Phys. Status Solidi B 176, 9-30 (1993).

${ }^{43}$ G. Bergmann, Phys. Rev. B 28, 515-522 (1983). 